\title{
How do Stability Corrections Perform in the Stable Boundary Layer Over Snow?
}

\author{
Sebastian Schlögl ${ }^{1,2}$ - Michael Lehning ${ }^{1,2}$ - Kouichi Nishimura ${ }^{3}$. \\ Hendrik Huwald ${ }^{2}$ - Nicolas J. Cullen ${ }^{4}$. Rebecca Mott ${ }^{1}$
}

Received: 27 February 2016 / Accepted: 11 May 2017 / Published online: 2 June 2017

C) Springer Science+Business Media Dordrecht 2017

\begin{abstract}
We assess sensible heat-flux parametrizations in stable conditions over snow surfaces by testing and developing stability correction functions for two alpine and two polar test sites. Five turbulence datasets are analyzed with respect to, (a) the validity of the Monin-Obukhov similarity theory, (b) the model performance of well-established stability corrections, and (c) the development of new univariate and multivariate stability corrections. Using a wide range of stability corrections reveals an overestimation of the turbulent sensible heat flux for high wind speeds and a generally poor performance of all investigated functions for large temperature differences between snow and the atmosphere above ( $>10 \mathrm{~K})$. Applying the Monin-Obukhov bulk formulation introduces a mean absolute error in the sensible heat flux of $6 \mathrm{~W} \mathrm{~m}^{-2}$ (compared with heat fluxes calculated directly from eddy covariance). The stability corrections produce an additional error between 1 and $5 \mathrm{~W} \mathrm{~m}^{-2}$, with the smallest error for published stability corrections found for the Holtslag scheme. We confirm from previous studies that stability corrections need improvements for large temperature differences and wind speeds, where sensible heat fluxes are distinctly overestimated. Under these atmospheric conditions our newly developed stability corrections slightly improve the model performance. However, the differences between stability corrections are typically small when compared to the residual error, which stems from the Monin-Obukhov bulk formulation.
\end{abstract}

Keywords Eddy-covariance method · Monin-Obukhov bulk formulation · Sensible heat flux $\cdot$ Snow $\cdot$ Stable boundary layer

Sebastian Schlögl

sebastian.schloegl@slf.ch

1 WSL-Institute for Snow and Avalanche Research SLF, Davos, Switzerland

2 School of Architecture, Civil and Environmental Engineering, École Polytechnique Fédérale de Lausanne, Lausanne, Switzerland

3 Graduate School of Environmental Studies, University of Nagoya, Nagoya, Japan

4 Department of Geography, University of Otago, Dunedin, New Zealand 


\section{Introduction}

The energy budget of a snow pack is mainly determined by net radiation and turbulent heat fluxes (Plüss and Mazzoni 1994), the latter contributing up to 50\% of the melt energy (Föhn 1973; Funk 1985; Pohl et al. 2006; Mott et al. 2011), especially in regions with generally high wind speeds. Modelling turbulent heat fluxes is a challenging task because atmospheric stability often has an influence that is significant but difficult to quantify. Based on the assumption of a constant-flux layer, Monin-Obukhov similarity theory (MOST, Obukhov 1946) leads to a bulk formulation of the turbulent heat fluxes (Blanc 1987). This technique has been deployed in almost all numerical models describing surface exchange, from climate research to the engineering of airplane wings.

Several studies (e.g. Cullen et al. 2007) investigated uncertainties in the bulk formulation and showed in general an acceptable agreement between eddy-covariance measurements and the bulk formulation over snow with some deficiencies caused by the violation of MOST assumptions:

1. For the stable boundary layer the applicability of the bulk formulation is limited to a certain range of the stability parameter $\zeta$ and the bulk Richardson number (e.g. Sharan and Kumar 2011).

2. The bulk formulation underestimates turbulent heat fluxes if measurements are made outside of the atmospheric surface layer (ASL) (Arck and Scherer 2002). Hence, the existence of constant fluxes is a mandatory assumption for the calculation of turbulent heat fluxes with the bulk formulation. This assumption ensures that the bulk fluxes are the same as the surface fluxes and is reasonably accurate in the ASL for near-neutral conditions $(0<\zeta<0.1)$ (Grachev et al. 2005).

3. Stationarity and horizontal homogeneity are further mandatory assumptions in the similarity theory, investigated by several studies in the 1980s (e.g. Joffre 1982; Högström 1988). These assumptions are typically strongly violated for test sites in complex terrain.

For Earth science applications, choice of an atmospheric stability correction is usually required as modelled turbulent heat fluxes assuming a neutral boundary layer tend to overestimate measured heat fluxes. Many different stability corrections for momentum $\psi_{m}$ and scalars $\psi_{s}$ have been published during the last few decades but show variable performance over snow (Andreas 2002). It has been noted that stability correction functions strongly depend on the chosen test site in such a way that no universal relation can be singled out as optimal (Martin and Lejeune 1998). Most well-established stability corrections depend exclusively on the stability parameter $\zeta$ (Dyer 1974 and references therein), with a few studies (e.g. Sorbjan 2010, 2016) developing stability corrections based on the bulk Richardson number. One novelty of our study is the development of a parametrization assuming a twoparameter dependence on buoyancy and shear terms in a first-order statistical model. To our knowledge, this is the first presentation of a stability correction that separates the bulk Richardson number into buoyancy and shear terms.

The main objective is therefore to assess stability corrections, in particular for stable conditions over snow in an effort to improve existing heat-flux parametrization schemes. We develop stability corrections in a purely empirical way, which is a well-known approach in the literature (e.g. Vickers et al. 2015). The sensitivity of turbulent heat-flux parametrizations has been shown to be largest for wind speeds between 3 and $5 \mathrm{~m} \mathrm{~s}^{-1}$ (Dadic et al. 2013). Therefore, we explicitly analyze the performance of different stability corrections as a function of atmospheric conditions. The paper is organized as follows: in Sects. 2-4 we introduce the selected datasets, the data processing methodology and the model set-up. In Sect. 5, the 
results are shown and discussed with respect to, (a) the applicability of MOST, (b) the model performance of well-established stability corrections and (c) the development of a univariate and a multivariate stability correction. Results are summarized in Sect. 6.

\section{Turbulence and Meteorological Data}

We used five different atmospheric turbulence datasets from four test sites, with these sites showing differences in their topographical characteristics. We chose one typical alpine test site with high topographical complexity (Weissfluhjoch, Davos, Switzerland) and three test sites consisting of one glacier site (Plaine Morte, Crans-Montana, Switzerland) and two polar sites (Greenland and Antarctica) representing a quasi-ideal site with homogeneous surface and quasi infinite fetch in all directions.

The turbulent sensible heat flux $(H)$ was calculated using the eddy-covariance method: $H=\rho c_{p} \overline{w^{\prime} \theta^{\prime}}$, where $\rho$ is the density of the air, $c_{p}$ is the heat capacity of the air, $w^{\prime}$ is the fluctuation of the vertical wind speed and $\theta^{\prime}$ is the virtual sonic temperature fluctuation. Note that the sonic temperature fluctuations have been converted into virtual temperature fluctuations.

Three-dimensional wind velocity and air temperature were processed using a linear detrending (Rannik and Vesala 1999) and a planar fit approach (Massmann and Lee 2002) to rotate the coordinate system. Air temperature, relative humidity and air pressure from weather stations were used to calculate air properties, which are required for the data processing. The weather stations are located in the immediate vicinity of the turbulence tower and are affected by the same air masses. Turbulence data were averaged to 30-min intervals, whilst changing to a 15-min time interval marginally affects the heat fluxes at the Weissfluhjoch test site (Mott et al. 2011). Note that we define a negative sensible heat flux as being directed towards the snow surface and a positive sensible heat flux as being directed upwards.

The selected datasets and corresponding test sites are summarized in Table 1 and briefly introduced below:

- Weissfluhjoch 2007 (WFJ07) A vertical set-up of two three-dimensional ultrasonic anemometers (CSAT3, Campbell Scientific, Inc.) was used at the traditional field site Weissfluhjoch (2540 $\mathrm{m}$ asl.) to measure three-dimensional wind velocity and air temperature at a frequency of $20 \mathrm{~Hz}$. The sensors were mounted 3 and $5 \mathrm{~m}$ above the ground and provided reliable data for 50 days between 11 February 2007 and 24 April 2007. Further information on the field campaign can be found in Stössel et al. (2010) and Mott et al. (2011).

Table 1 Overview of the different datasets

\begin{tabular}{llccl}
\hline Area & Date & Number of days & Frequency $(\mathrm{Hz})$ & Level above ground $(\mathrm{m})$ \\
\hline WFJ07 & Feb-Apr 2007 & 50 & 20 & 3,5 \\
WFJ11 & Feb+Mar, 2011-13 & 169 & 10 & 5 \\
PM07 & Feb-Apr 2007 & 37 & 20 & 3.75 \\
GR00 & Jun 2000-01 & 16 & 50 & 1,2 \\
AA00 & Oct-Nov 2000 & 43 & 100 & $0.2,1,25$
\end{tabular}

The number of analyzed days, the frequency of the sonic anemometer and the vertical level above the ground are shown 
- Weissfluhjoch 2011-13 (WFJ11) Three-dimensional wind velocity and air temperature were recorded at $5 \mathrm{~m}$ above the ground at a frequency of $10 \mathrm{~Hz}$ with a three-dimensional ultrasonic anemometer (CSAT3). The analysis was conducted for data obtained between February and March in the years 2011-13.

- Plaine Morte 2007 (PM07) Two three-dimensional ultrasonic anemometers (CSAT3) were installed on a horizontal boom facing opposite directions (west-north-west vs. east-southeast) at $3.75 \mathrm{~m}$ above the ground to measure air temperature and three-dimensional wind velocity at $20 \mathrm{~Hz}$. The data were collected at the almost flat field site on the Plaine Morte glacier (2750 m asl.) near Crans-Montana, Switzerland from February to April 2007. High quality meteorological data were additionally recorded and used to force the model. A detailed description about the set-up at the Plaine Morte glacier can be found in Huwald et al. (2009) and Bou-Zeid et al. (2010).

- Greenland 2000 (GRO0) High-frequency three-dimensional ultrasonic anemometer measurements (CSAT3) were recorded at $50 \mathrm{~Hz}$ at the Summit Camp $\left(72.3^{\circ} \mathrm{N}, 38.8^{\circ} \mathrm{W}\right.$, $3208 \mathrm{~m}$ asl.) located on the northern dome of the Greenland ice sheet. Data were collected at $1 \mathrm{~m}$ and $2 \mathrm{~m}$ above the snow surface during summer in 2000 and 2001 . Additionally, meteorological measurements were obtained for the post processing and used to force the model. More information about the field campaign can be found in Cullen et al. (2007, 2014).

- Antarctica 2000 (AA00) A set-up of three vertical three-dimensional ultrasonic anemometers (DA-600, Kaijo Denki) were installed at Mizuho Station $\left(70^{\circ} 42^{\prime} \mathrm{S}, 44^{\circ} 20^{\prime} \mathrm{E}\right.$, $2230 \mathrm{~m}$ asl.) in Eastern Antarctica at 0.2, 1 and $25 \mathrm{~m}$ and recorded turbulence data at a frequency of $100 \mathrm{~Hz}$ from October to November 2000. Longwave and shortwave radiation, relative humidity, air and snow surface temperature were additionally measured and used to force the model. More information about the field campaign can be found in Nishimura and Nemoto (2005).

\section{Model}

We calculated surface turbulent heat fluxes using the physically-based model SNOWPACK (Lehning et al. 2002). Meteorological data (air and snow surface temperature, relative humidity, incoming and outgoing shortwave and longwave radiation) were required to force the model and taken from weather stations or meteorological sensors close to the turbulence towers. Wind velocities were used directly from the three-dimensional ultrasonic anemometers and sensible heat fluxes calculated using Monin-Obukhov bulk formulation,

$$
H=\rho c_{p} C_{H} \bar{U} \Delta \theta
$$

where $\Delta \theta=\theta_{s}-\theta_{z_{\text {ref }}}$ is the virtual potential temperature difference, $\theta_{z_{\text {ref }}}$ is the virtual potential temperature at the reference height, $\theta_{S}$ is the virtual potential temperature at the snow surface, $\bar{U}$ is the mean wind speed and $C_{H}$ is the exchange coefficient for stable conditions,

$$
C_{H}=\frac{k^{2}}{\left[\ln \left(\frac{z_{\text {ref }}}{z_{0 M}}\right)-\psi_{m}(\zeta)\right]\left[\ln \left(\frac{z_{\text {ref }}}{z_{0 M}}\right)-\psi_{s}(\zeta)\right]},
$$

where $k=0.4$ is the von Kármán constant, $\zeta=\left(-k z_{\text {ref }} g T_{*}\right) /\left(\theta_{s} u_{*}^{2}\right)$ is the modelled stability parameter (stability parameter henceforth), $u_{*}=k \bar{U}\left(\ln \left(z_{\text {ref }} / z_{0 M}\right)-\psi_{m}\right)^{-1}$ is the modelled friction velocity, $T_{*}=k\left(\theta_{s}-\theta_{z_{\text {ref }}}\right)\left(\ln \left(z_{\text {ref }} / z_{0 M}\right)-\psi_{s}\right)^{-1}$ is the modelled 
temperature scale, $z_{0 M}$ is the aerodynamic roughness length and $\psi_{m}$ and $\psi_{s}$ are the stability corrections for momentum and scalars. In our analysis, we used the simple approach that the roughness lengths for momentum and scalars are equal $\left(z_{0 M}=z_{0 T}\right)$ although several studies (Andreas 1987; Andreas et al. 2010; Smeets and van den Broeke 2008b; Conway and Cullen 2016) suggest $z_{0 M} \gg z_{0 T}$ in the case of fully rough flow in agreement with surface renewal theory. An analysis of the parametrization of the scalar roughness length $z_{0 T}$ is beyond the scope of the present study, though we note that assuming a separate scalar roughness length does not significantly improve the heat-flux parametrization (not shown).

\section{Methods}

We focus on meteorological conditions excluding the following situations:

1. Patchy snow cover: turbulent heat fluxes over a patchy snow cover are extremely variable and difficult to quantify as the advective heat transport significantly alters the boundarylayer characteristics (Essery et al. 2006; Mott et al. 2013, 2015).

2. Heavy precipitation: turbulence measurements are not reliable during heavy precipitation because snowflakes/raindrops passing through the three-dimensional ultrasonic sampling volume disturb the measurement.

3. An unstable boundary layer: unstable conditions above snow can occur, e.g. in summer over dry snow (Cullen and Steffen 2001), but are generally rather infrequent. Herein we focus only on stability corrections for stable conditions.

The following analysis steps have been conducted for the different turbulence datasets:

\subsection{Assessment of Monin-Obukhov Validity by Applying a Non-parametrized $\psi$ Function}

An estimation of the non-parametrized stability correction based on the measured heat-flux values was conducted. This separates the error due to the bulk formulation from that introduced through the parametrizations of the correction functions. Measured friction velocities $\left(u_{* \text {,meas }}\right)$ and temperature scales $\left(T_{*, \text { meas }}\right)$ from the three-dimensional ultrasonic anemometers are required to calculate non-parametrized stability correction values for momentum $\psi_{m}$ and heat $\psi_{s}$,

$$
\begin{aligned}
& \psi_{m}=\ln \left(\frac{z_{\text {ref }}}{z_{0 M}}\right)-\frac{k \bar{U}}{u_{*, \text { meas }}}, \\
& \psi_{s}=\ln \left(\frac{z_{\text {ref }}}{z_{0 M}}\right)-\frac{k \Delta \theta}{T_{*, \text { meas }}} .
\end{aligned}
$$

Note that changes to the measurement height $z_{\text {ref }}$ due to a changing snow depth are taken into account.

\subsection{Characterization of Well-Established Stability Corrections}

We tested six different stability corrections (Stearns and Weidner 1993; modified by Michlmayr et al. 2008; Holtslag and De Bruin 1988; Beljaars and Holtslag 1991; Grachev et al. 2007 and a log-linear expression) with the SNOWPACK model and compared them against the assumption of a neutral atmosphere (see Table 6 in the Appendix). The six investigated stability parametrizations are exclusively dependent on the stability parameter $\zeta$, which 
implies that all relevant information is found in one variable. The original Stearns and Weidner stability correction (original Stearns henceforth) for momentum recommends logarithmic and inverse tangent terms of the fourth root of $\zeta$ and differs only slightly for the stability correction for heat and was developed from measurements in Antarctica. The modified Stearns and Weidner stability correction (modified Stearns henceforth) contains the same functional form as the original stability correction of Stearns with different empirical coefficients. The original Stearns stability correction is recommended for low air temperatures (Stearns and Weidner 1993). The stability correction of Holtslag and de Bruin (1988; Holtslag henceforth) for heat and momentum is identical and consists of linear and exponential terms with five empirical coefficients. This stability correction is recommended for very stable conditions (Andreas 2002). The stability correction of Beljaars and Holtslag(1991; Beljaars henceforth) differs for heat and momentum. This stability correction for momentum contains the same functional form as Holtslag's stability correction with different empirical coefficients. Beljaars' stability correction for heat differs from those of Holtslag in one functional term. Holtslag's and Beljaars' stability corrections were both derived over a grass-covered surface at Cabauw, Netherlands. Grachev et al. (2007) proposed stability corrections developed from measurements in the Arctic separately for momentum and heat.

The stability correction using the simple log-linear expression is based on theoretical suggestions for weakly stable conditions (e.g. Munro 1980) and exclusively consists of one empirical coefficient $\beta$, which varies between a value of 4 (Webb 1970) and 7 (Large and Pond 1982; Högström 1988). We chose $\beta=5$ for the stability correction for momentum and heat in our assessment, which is the coefficient used in the energy-balance snow model SNOBAL (Marks and Dozier 1992). A further variant of correction, used in the snow modelling community, e.g. in the community land model (CLM) (Zeng et al. 1998) or in the joint UK land environment simulator (JULES) investigation model (JIM) (Essery et al. 2013), distinguishes between near-neutral and stable cases. The stability corrections in the landsurface models CLM or JIM and in the energy-balance snow model SNOBAL are identical for near-neutral cases and slightly differ for stable conditions. A comparison between both models indicates no significant improvement of the performance of the sensible heat fluxes in the CLM or JIM models (not shown).

We additionally separated our high quality data into sub-critical and supercritical cases according to Grachev et al. (2013) in order to account for the applicability of MOST in stable conditions. The gradient Richardson number $R i=g \theta^{-1}(d \theta / d z)(d \bar{U} / d z)^{-2}$ and flux Richardson number $R_{f}=-g \theta^{-1} \overline{w^{\prime} \theta^{\prime}} u_{*}^{-2}(d \bar{U} / d z)^{-1}$ have been calculated for this purpose. Data points were separated in the turbulent part of the flow (sub-critical cases: $R i<R i_{c r}=0.2$ and $R_{f}<R_{f, \text { cr }}=0.2$ ) and the laminar part of the flow (supercritical cases: $R i>R i_{\text {cr }}=0.2$ and $R_{f}>R_{f, \mathrm{cr}}=0.2$ ). Expressing these regimes in terms of the stability parameter, the flow is turbulent for $\zeta<1$ and laminar for $\zeta>1$ by assuming $\beta=5$ (see above). Note that a hysteresis effect may lead to a turbulent flow regime until $R i=1$ or $\zeta=5$, if the flow was previously turbulent. The applicability of MOST is theoretically limited to sub-critical cases.

\subsection{Development of a Simple Alternative Model}

We developed test-site specific parametrizations and assessed a test-site independent, universal parametrization based on a randomly chosen subset of $67 \%$ of the individual datasets (Sects. 5.3 and 5.4). The remaining $33 \%$ of the individual datasets were used for a validation and comparison between our newly developed parametrizations and the well-established 
parametrizations (Sect. 5.5). The coefficients of the universal parametrization are calculated based on a combined large dataset from all experiments. Additionally, all datasets were artificially replicated to reach the size of the largest dataset (WFJ11) due to the inhomogeneity of the size of separate datasets. This method is more robust than averaging the coefficients of the test-site-specific parametrizations.

Based on our high quality datasets we developed two alternative approaches: (a) a univariate parametrization assuming a conventional linear dependence on the measured stability parameter $\zeta_{\text {meas }}=z_{\text {ref }} L^{-1}$ where $L=-u_{* \text {,meas }}^{3} \theta / k g \overline{w^{\prime} \theta^{\prime}}$ is the Obukhov length, and (b) a multivariate parametrization assuming a dependence on buoyancy and shear terms in a firstorder statistical model. The development of both approaches was conducted for conditions in a stable atmosphere $\left(0<\zeta_{\text {meas }}<10\right)$.

(a) To avoid a large number of empirical coefficients, we decided to choose a linear functional form for the stability correction. This reduces the number of empirical coefficients to a minimum,

$$
\begin{aligned}
\psi_{m} & =m_{1} \zeta_{\text {meas }}, \\
\psi_{s} & =m_{2} \zeta_{\text {meas }},
\end{aligned}
$$

where $m_{1}$ and $m_{2}$ are dimensionless empirical coefficients, which are negative in the case of stable stratification.

In order to estimate the empirical coefficients, the non-parametrized $\psi$ values (Eqs. 3 and 4) were plotted as a function of the measured stability parameter, and due to large scatter in the datasets we decided to conduct a logarithmic bin-averaging of the data. We chose logarithmic bin-averaging instead of equally-spaced bin-averaging as the frequency of nearneutral atmospheric conditions is large and decreases exponentially with increasing stability. We selected the median instead of the mean for averaging the $\psi$ values because the median is less sensitive to outliers and leads to more robust results. Finally, the linear regression was conducted with a least-squares scheme.

Additionally, we tested polynomials of different orders to find the optimum agreement and highest regression coefficient. While the optimum agreement significantly changed between the chosen test sites, a linear regression with an offset $b$ showed the highest correlation coefficient for most cases. However, in the following, we explicitly exclude a potential offset $b$ because any offset would preclude recovering the asymptotic behaviour towards neutral conditions correctly. Additionally, exponential functions and other dependencies were analyzed but gave inconsistent results and are therefore not discussed further.

(b) The bulk Richardson number is separated into non-dimensional buoyancy $B=\Delta T / \bar{T}$ ( $\bar{T}$ is the mean of the air $(T)$ and snow surface temperature $\left(T_{s n}\right)$ ) and shear contributions $S=z_{\text {ref }} g / \bar{U}^{2}$. This relationship is used to conduct a second parametrization assuming a two-parameter dependence on buoyancy and shear terms in a first-order statistical model,

$$
\begin{aligned}
\psi_{m}\left(T, T_{s n}, \bar{U}\right) & =a_{1} B+b_{1} S, \\
\psi_{s}\left(T, T_{s n}, \bar{U}\right) & =a_{2} B+b_{2} S,
\end{aligned}
$$

where $a_{1}, a_{2}, b_{1}$ and $b_{2}$ are the dimensionless empirical coefficients. The purpose of this test model is to simply determine whether undesirable effects due to combining shear and buoyancy into one term can be alleviated.

Statistical models of second or higher order were also evaluated, and we found no sufficient improvement of $R^{2}$, which leads to the presentation of a first-order statistical model only. We additionally tested statistical models with an offset and discuss the model performance in Sect. 5.4. 


\section{Results and Discussion}

\subsection{The Uncertainty of the Bulk Formulation}

We investigated differences between the eddy-covariance method and the bulk formulation and assessed the uncertainty occurring through application of the bulk formulation. This uncertainty in heat-flux parametrization is interpreted as the minimum error and defines the limitation of bulk formulations. The mean absolute error $(M A E)$ due to the parametrization is on average $6 \mathrm{~W} \mathrm{~m}^{-2}$ (Table 3 ). The approach slightly underestimates measured sensible heat fluxes, quantitatively estimated by using the mean bias error $\left(M B E=1 \mathrm{~W} \mathrm{~m}^{-2}\right)$. A comprehensive evaluation has shown that small sensible heat fluxes $\left(\leq 30 \mathrm{~W} \mathrm{~m}^{-2}\right)$ towards the snow surface are satisfactorily simulated with the bulk formulation, whereas larger heat fluxes are underestimated significantly (Fig. 1) in agreement with Guo et al. (2011).

Two opposite effects explain these differences: the bulk formulation underestimates heat fluxes for large temperature differences, while on the other hand, heat fluxes are overestimated for wind speeds $>2 \mathrm{~ms}^{-1}$. This observation has a strong ramification for simulating turbulent heat fluxes for the Antarctic test site, where data reveal a mean wind speed of $10 \mathrm{~m} \mathrm{~s}^{-1}$. Despite near-neutral atmospheric conditions, large wind speeds lead to modelled sensible heat fluxes up to $100 \mathrm{~W} \mathrm{~m}^{-2}$. In contrast, results from the eddy-covariance method show only small sensible heat fluxes up to $10 \mathrm{~W} \mathrm{~m}^{-2}$. An uncertainty in the snow surface temperature of $1 \mathrm{~K}$ actually results in a modelled sensible heat flux uncertainty of up to $50 \mathrm{~W} \mathrm{~m}^{-2}$ for the meteorological conditions in Antarctica, and this uncertainty distinctly decreases with decreasing wind speeds and increasing temperature difference. We recommend careful attention when analyzing turbulent sensible heat fluxes in the presence of large wind speeds and small temperature differences. Because of the high sensitivity to the snow surface temperatures for conditions found in

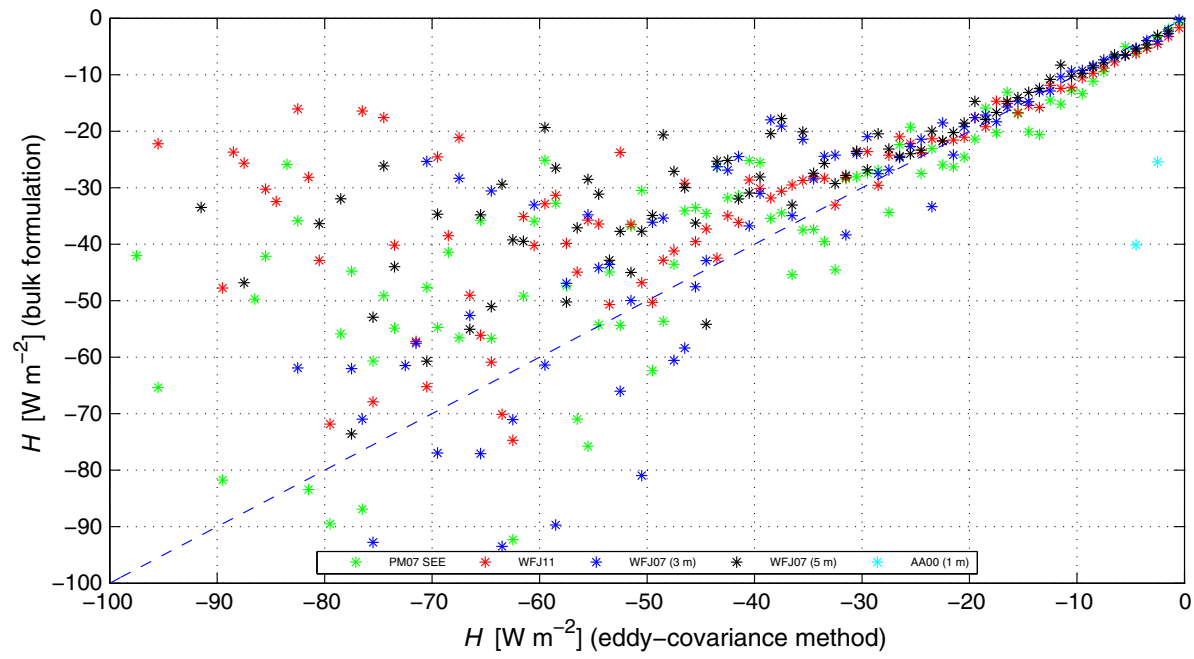

Fig. 130 -min sensible heat fluxes $\left[\mathrm{W} \mathrm{m}^{-2}\right]$ : Measurements processed with the eddy-covariance method $(x$-axis) and model results calculated with the bulk formulation ( $y$-axis) are shown for PM07 SEE (green), WFJ11 (red), WFJ07 (3 m) (blue), WFJ07 (5 m) (black) and AA00 (1 m) (cyan) 
Antarctica, we decided to develop the stability corrections without using the Antarctica dataset.

In summary, uncertainties in the bulk formulation may be due to the violation of the necessary assumptions for using MOST. For example, our investigations show that the assumption of a constant-flux layer (difference of sensible heat fluxes of two different vertical measurement level is smaller than $10 \%$, see Stull 1988) is only valid $23 \%$ of the time for the GR00 dataset and only $10 \%$ of the time at the Weissfluhjoch test site by comparing measured turbulent sensible heat fluxes at two vertical levels. The MOST assumption of an ideal test site with a quasi-infinite fetch in all directions is only fulfilled for the Greenland test site, and for test sites in complex terrain mean absolute errors are distinctly larger than for Greenland.

\subsection{Evaluation of Well-Established Stability Corrections}

Applying different stability corrections clearly affects the absolute values of the sensible heat flux. The resulting MAE values (Table 3) show the optimum model performance of the sensible heat flux for the Holtslag, Beljaars and original Stearns stability corrections $\left(M A E: 7 \mathrm{~W} \mathrm{~m}^{-2}\right)$, followed by the log-linear assumption $\left(M A E: 8 \mathrm{~W} \mathrm{~m}^{-2}\right)$, Grachev et al. (2007) $\left(M A E: 11 \mathrm{~W} \mathrm{~m}^{-2}\right)$ and the modified correction of Stearns $\left(M A E: 11 \mathrm{~W} \mathrm{~m}^{-2}\right)$. It is remarkable that the simple log-linear stability correction performs better than the modified correction of Stearns. The assumption of a neutral boundary layer shows the largest $M A E$ values $\left(M A E: 22 \mathrm{~W} \mathrm{~m}^{-2}\right)$ and should not be used when modelling turbulent sensible heat fluxes in stable conditions. These findings are in agreement with, e.g., Dadic et al. (2013). Note that for the WFJ11 and the GR00 (2 m) datasets some stability corrections provide even better results than the non-parametrized $\psi$ function (Sect. 5.1), which could be interpreted as error compensation (Table 2).

Additionally, the mean bias error $(M B E)$ is calculated to indicate a systematic model error by applying different stability corrections (Table 3). Modified Stearns and the neutral boundary layer overestimate the turbulent sensible heat flux, whereas the Holtslag, Beljaars and Grachev stability corrections, the original Stearns stability correction, and the log-linear approach tend to underestimate the heat fluxes.

The results of the $M A E$ values are consistent for the individual test sites with a highly variable magnitude of $M A E$ values depending on the test site. The larger $M A E$ values in the PM07 dataset may be the result of katabatic flows, which may lead to a larger deviation from idealized conditions.

The performance of sensible heat-flux parametrizations strongly depends on atmospheric conditions. Therefore, as an example we investigated the quality of model results as a function of the air temperature $T$, the temperature difference $\Delta T$, the wind speed $\bar{U}$ and the stability parameter $\zeta$, shown for the stability correction of Holtslag (Fig. 2) but also valid for the other investigated stability corrections.

The performance of sensible heat-flux parametrizations is not distinctly dependent on air temperature but decreases with an increase in wind speed and temperature difference as discussed above. Stability corrections perform reasonably well for a small temperature difference. In other words, stability corrections need improvements in very stable conditions and perform satisfactorily in near-neutral conditions where only a small correction is required.

Depending on the chosen test site, $50-70 \%$ of the data consist of sub-critical cases. Sensible heat fluxes are modelled more accurately for the sub-critical cases shown for the WFJ11 dataset (Table 3); MAE values decrease by $2 \mathrm{~W} \mathrm{~m}^{-2}$ on average when considering only 


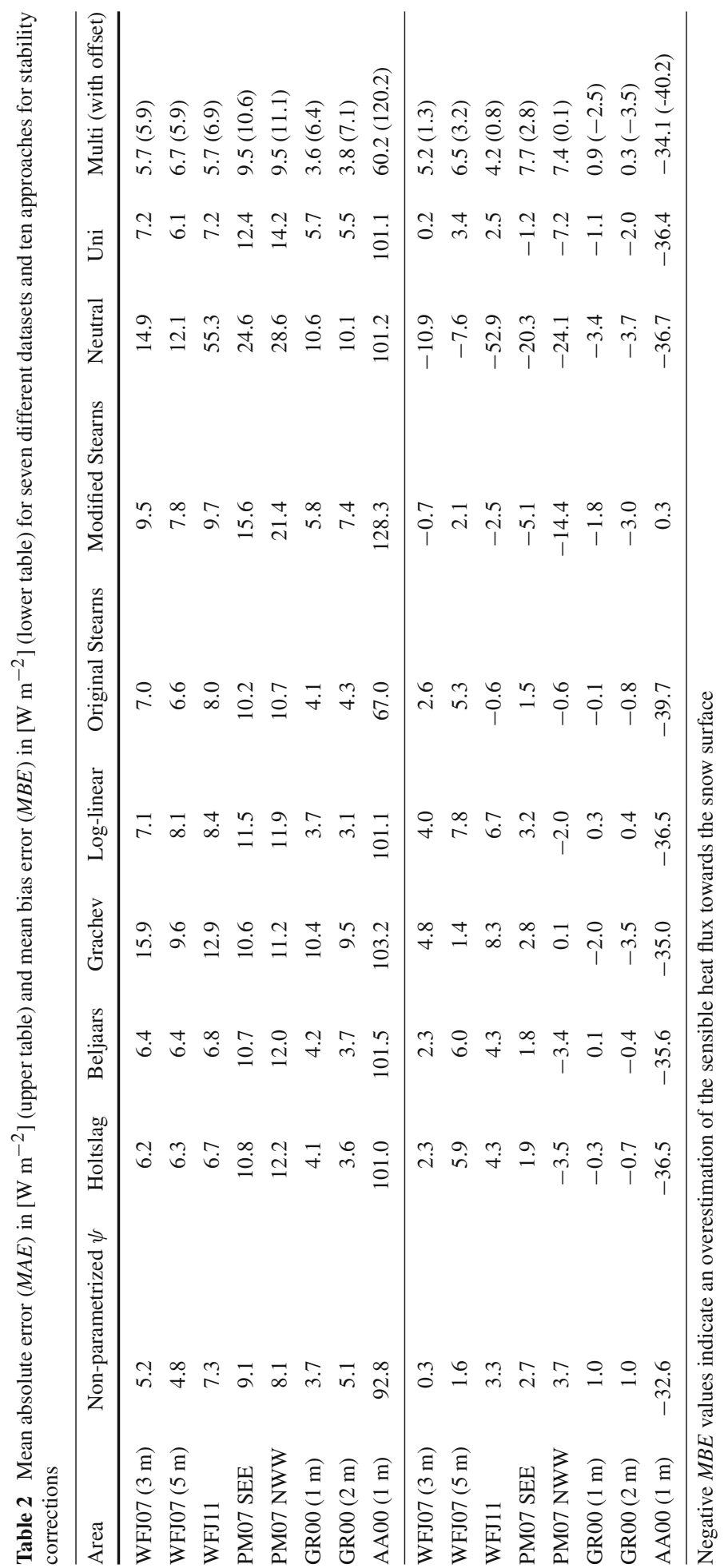


Table 3 Mean absolute error $(M A E)$ in $\left[\mathrm{W} \mathrm{m}^{-2}\right]$ (upper table) and mean bias error $(M B E)$ in $\left[\mathrm{W} \mathrm{m}^{-2}\right.$ ] (lower table) for the WFJ11 dataset and five approaches for stability corrections

Negative $M B E$ values indicate an overestimation of the sensible heat flux towards the snow surface. Data were separated in sub-critical cases, supercritical cases and all cases (same values as Table 2)

\begin{tabular}{lccr}
\hline MAE & Sub-critical & Supercritical & \multicolumn{1}{c}{ All } \\
\hline Modified Stearns & 8.2 & 11.3 & 9.7 \\
Original Stearns & 6.1 & 10.0 & 8.0 \\
Holtslag & 6.2 & 7.0 & 6.7 \\
Log-linear & 7.4 & 9.1 & 8.4 \\
Neutral & 37.4 & 70.4 & 55.3 \\
\hline MBE & Sub-critical & Supercritical & All \\
\hline Modified Stearns & -2.0 & -2.8 & -2.5 \\
Original Stearns & 0.4 & -2.3 & -0.6 \\
Holtslag & 2.9 & 5.0 & 4.3 \\
Log-linear & 4.8 & 7.7 & 6.7 \\
Neutral & -35.2 & -68.0 & -52.9 \\
\hline
\end{tabular}
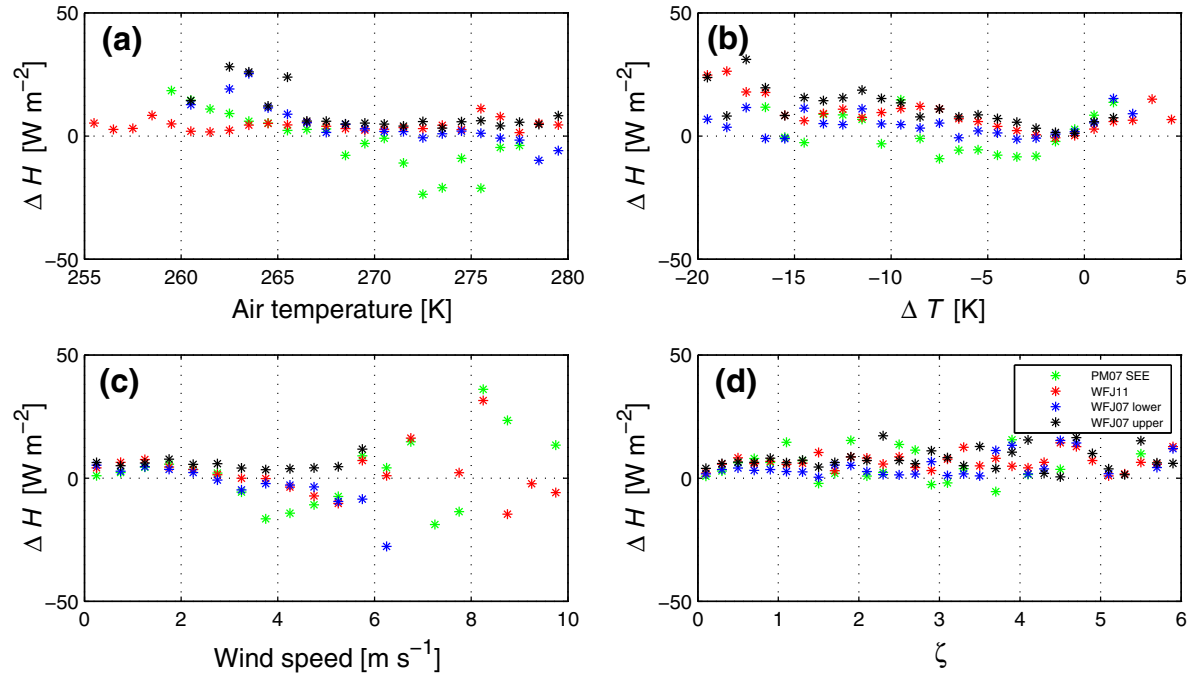

Fig. 2 Differences between modelled (Holtslag) and measured sensible heat flux [W $\left.\mathrm{m}^{-2}\right]$. The differences are shown as functions of air temperature $T$, temperature difference $\Delta T$, wind speed $\bar{U}$ and stability parameter $\zeta$. The test sites WFJ11 (red), WFJ07 lower (blue), WFJ07 upper (black) and PM07 SEE (green) were selected for the visualization

sub-critical cases. Additionally we found an improvement in $M B E$ values. The improved representation of sensible heat fluxes in the sub-critical cases is caused by the fact that MOST is only applicable in this range and the trivial fact that sensible heat fluxes are on average larger in the supercritical cases, which causes in general larger absolute errors. However, we note that in model applications, e.g. model simulations of the seasonal snow cover, the supercritical cases need to be covered, too. Since to our knowledge no generally accepted or widely used alternative exists to simply extending the MOST parametrization, the values as presented in Table 2 are of practical validity. 

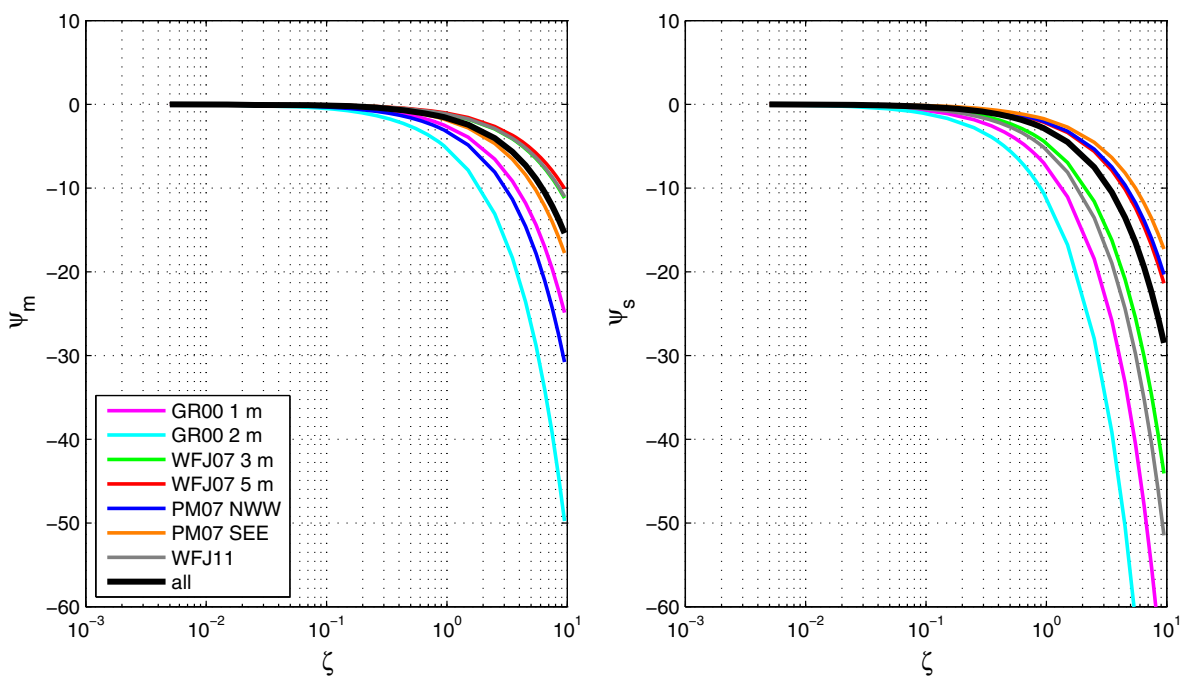

Fig. 3 Stability correction functions $\psi_{m}$ and $\psi_{s}$ as a function of the measured stability parameter $\zeta$ for the different test sites separately and the universal parametrization (black line)

\subsection{Parametrization for a Stability Correction Based on the Measured Stability Parameter (Univariate Parametrization)}

To test how far the functional forms of established stability corrections provide extra value to our datasets, we developed a simple linear regression of the correction function with the measured stability parameter as an independent variable. As a first step, correction functions have been developed for the individual sites; coefficients of the parametrization strongly depend on the chosen test site and show a large spread in magnitude (Fig. 3).

The largest stability correction for momentum was found for the GR00 (2 m) dataset $\left(m_{1}=-5.24\right)$, whereas the lowest correction is required for the WFJ07 $(5 \mathrm{~m})$ dataset $\left(m_{1}=-1.06\right)$. The spread for the test-site-specific stability corrections for scalars is larger and shows again the largest stability corrections for the Greenland test site and the lowest stability corrections for Weissfluhjoch. Hence, the stability correction for the quasi-ideal test site Greenland is large and decreases with increasing complexity of the topography. These findings need to be tested for more stations of different complexity to confirm this result.

The substantial dependence upon the test site has already been observed by Martin and Lejeune (1998) and is clearly confirmed herein. However, it is desirable to find a universal stability correction for terrain of different complexity covered in snow. As described in Sect. 4.3, we therefore also derived an overall model, and for the universal parametrization we estimated $m_{1}=-1.63$ and $m_{2}=-2.96$.

$M A E$ values of this universal univariate parametrization, solely dependent on the stability parameter, amount on average to $7 \mathrm{~W} \mathrm{~m}^{-2}$. This $M A E$ value is split into a $6 \mathrm{~W} \mathrm{~m}^{-2}$ uncertainty due to the bulk formulation and additionally $1 \mathrm{~W} \mathrm{~m}^{-2}$ uncertainty by applying the parametrization. One exception holds for the Greenland ice sheet, and for this test site, the univariate parametrization performs better than the non-parametrized $\psi$ values. In general, the univariate parametrization shows a systematic bias of $2 \mathrm{~W} \mathrm{~m}^{-2}$. Compared with the well-established stability corrections we improved the model performance for moderate wind speeds (2-5 $\mathrm{m} \mathrm{s}^{-1}$ ) (Fig. 4). 

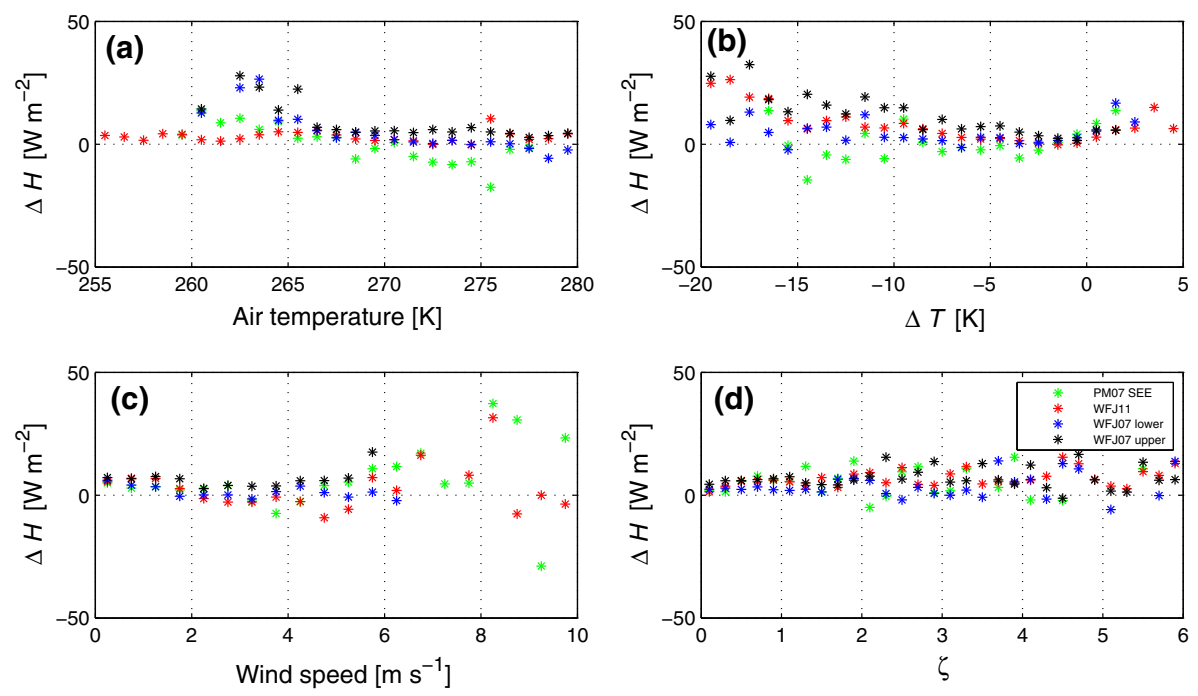

Fig. 4 Differences between modelled and measured sensible heat flux [W $\left.\mathrm{m}^{-2}\right]$ for the univariate parametrization. The differences are shown as functions of air temperature, temperature difference, wind speed and stability parameter. WFJ11 (red), WFJ07 lower (blue), WFJ07 upper (black) and PM07 SEE (green) were selected for the visualization

\subsection{Parametrization of the Stability Correction Based on Buoyancy and Shear Terms Separately (Multivariate Parametrization)}

The stability parameter represents the balance between buoyancy and shear term as a simple ratio, having both desirable properties but also mathematical problems for low wind speeds. We therefore test an independent treatment of buoyancy and shear terms in a linear model as a stability correction (Eqs. 6a, 6b). The $M A E$ values of the universal parametrization for this approach are on average $7 \mathrm{~W} \mathrm{~m}^{-2}$, with the multivariate parametrization having a systematic bias of $4 \mathrm{~W} \mathrm{~m}^{-2}$. In summary the multivariate parametrization performs slightly worse than the univariate parametrization, confirming that the combination of buoyancy and shear terms is an acceptable choice.

Coefficients of the multivariate parametrization vary by one order of magnitude for the different test sites and are therefore shown to be highly sensitive to test-site-specific settings (Table 4).

The multivariate parametrization still tends to underestimate measured sensible heat fluxes, especially for large temperature differences and high wind speeds. However in terms of $M A E$, the universal multivariate parametrization performs better than all investigated stability corrections, especially for high wind speeds. A second advantage of the multivariate parametrization is related to the number of model uncertainties.

Physically-based models usually calculate the stability parameter for MOST in order to determine surface turbulent heat fluxes, and investigations have shown a large discrepancy between modelled and measured stability parameters. The modelled stability parameter tends to have larger values especially for low wind speeds and large temperature differences (not shown). These uncertainties directly affect stability corrections and hence modelled surface fluxes, where we found up to $20 \mathrm{~W} \mathrm{~m}^{-2}$ smaller sensible heat fluxes by using the modelled stability parameter instead of the measured stability parameter. The approach of the multivari- 
Table 4 Dimensionless empirical coefficients of the multivariate parametrization for $\psi_{m}$ (left) and $\psi_{s}$ (right) for different test sites

\begin{tabular}{lrrlrr}
\hline test site & \multicolumn{1}{l}{$a_{1}$} & $b_{1}$ & test site & \multicolumn{1}{c}{$a_{2}$} & \multicolumn{1}{c}{$b_{2}$} \\
\hline WFJ07 $(3 \mathrm{~m})$ & 3.227 & 0.0043 & WFJ07 $(3 \mathrm{~m})$ & -982.90 & -0.0005 \\
WFJ07 $(5 \mathrm{~m})$ & -4.441 & 0.0025 & WFJ07 $(5 \mathrm{~m})$ & -642.51 & 0.0009 \\
WFJ11 & -30.74 & 0.0008 & WFJ11 & -1135.4 & -0.0015 \\
PM07 NWW & -191.93 & 0.0008 & PM07 NWW & -751.73 & -0.0005 \\
PM07 SEE & -29.55 & 0.0090 & PM07 SEE & -692.74 & -0.0123 \\
GR00 (1 m) & -145.41 & -0.0914 & GR00 (1 m) & -378.92 & -2.0489 \\
GR00 (2 m) & -179.56 & -0.0369 & GR00 (2 m) & -243.93 & -0.7448 \\
Universal & -65.35 & 0.0017 & Universal & -813.21 & -0.0014 \\
\hline
\end{tabular}

ate stability correction is independent of the stability parameter and eliminates the uncertainty in the modelled stability parameter. Related to this aspect, we recommend the application of a multivariate stability correction on the condition that high quality meteorological variables are also recorded.

Including an offset $c_{1}$ and $c_{2}$ (in Eqs. $6 \mathrm{a}$ and $6 \mathrm{~b}$ ) lowers the systematic bias of $4.6 \mathrm{~W} \mathrm{~m}^{-2}$ to $0.3 \mathrm{~W} \mathrm{~m}^{-2}$ and leads to a much better representation of the sensible heat fluxes. Investigations have shown that the offset for the stability correction of momentum is almost zero $\left(c_{1}=\right.$ -0.69 ), but for the stability correction of heat we found an offset $c_{2}=6.73$. This offset can be interpreted as the offset $\ln \left(z_{\text {ref }} / z_{0}\right)$ in Eq. 4, which is valid for neutral conditions with no temperature difference. Results of the multivariate stability correction with offset are shown in Tables 2 and 5 in brackets.

\subsection{Validation}

In order to compare our newly developed parametrizations with well-established stability corrections, we used the remaining one-third of the datasets (not used for the parametrization development) for validation. Since the performances of stability corrections were shown to be highly sensitive to the chosen test site, we analyze the average $M A E$ and $M B E$ values of all datasets for the model performance test, considering a large range of air temperatures, temperature differences and wind speeds (Table 5).

Table $5 M A E\left[\mathrm{~W} \mathrm{~m}^{-2}\right]$ and $M B E\left[\mathrm{~W} \mathrm{~m}^{-2}\right]$ values averaged over the five different datasets for six well-established stability corrections, the assumption of a neutral boundary layer and the newly developed parametrizations

\begin{tabular}{lll}
\hline Stability correction & $M A E$ & $M B E$ \\
\hline Holtslag & 7.0 & 1.5 \\
Beljaars & 7.2 & 1.5 \\
Grachev & 11.4 & 1.7 \\
Modified Stearns & 10.9 & -3.6 \\
Original Stearns & 7.1 & 1.1 \\
Log-linear & 7.6 & 3.0 \\
Neutral & 21.9 & -17.5 \\
Univariate & 6.7 & 2.0 \\
Multivariate (with offset) & $6.4(7.7)$ & $4.6(0.3)$
\end{tabular}


The consideration of both errors $(M A E$ and $M B E)$ is required to allow a comprehensive analysis of the performance of the parametrizations. Stability corrections due to Holtslag, Beljaars, original Stearns, log-linear and our two newly developed parametrizations have a similar $M A E$ value of $7 \mathrm{~W} \mathrm{~m}^{-2}$ on average and slightly underestimate the turbulent heat fluxes. The modified Stearns parametrization and the neutral boundary-layer assumption overestimate the heat fluxes at the measurement height. Note that modified Stearns was introduced by Michlmayr et al. (2008) in order to allow a more accurate simulation of snow-surface dynamics, in particular snow-surface temperature. Further investigations are required to assess how far near-surface flux divergence versus snow-model inaccuracies are responsible for surface temperatures being better simulated with modified Stearns but local heat fluxes at the measurement height are not more accurate. The multivariate stability correction with offset (shown in brackets) shows almost no systematic bias.

Additionally, spatial cross validations have been conducted for all datasets by using the test-site-specific parametrizations and the universal parametrization. As expected, the smallest $M A E$ value averaged over the five different datasets was found for the test-sitespecific parametrizations, which correspond to the related test sites. The test-site-specific parametrizations, developed from other test sites, provide similarly reliable results. The $M A E$ value is merely increased by $1 \mathrm{~W} \mathrm{~m}^{-2}$ using parametrizations of other test sites, and the universal parametrization performs slightly better than the parametrizations of other test sites, but worse than the parametrizations of the same test site. In terms of $M B E$ values we analyzed the lowest systematical bias for the test-site-specific parametrizations, which correspond to the related test sites and a $1 \mathrm{~W} \mathrm{~m}^{-2}$ larger systematical bias for the universal parametrization and the test-site-specific parametrizations from other test sites.

Analyzing seasonal dependencies, we investigated temporal cross validations for the WFJ11 dataset for March 2012. For this experiment, coefficients for the parametrizations were developed based on monthly subsets of the entire WFJ11 dataset. In general, we analyzed no seasonal dependence and a similar $M A E$ value with a difference of less than $1 \mathrm{~W} \mathrm{~m}^{-2}$ for parametrizations developed for different months.

Results from the spatial and temporal cross validations hold for the univariate and multivariate parametrizations and reveal that the choice of a test-site-specific parametrization is not crucial for the performance of heat-flux parametrizations. Finally, these results show the robustness of the developed stability corrections that are applicable for snow-covered terrain. Further it legitimises the creation of a universal parametrization developed from all five datasets.

\subsection{Model Performance as a Function of Meteorological Conditions}

We compare the performance of the nine different stability corrections against a range of typical atmospheric conditions for the WFJ11 validation dataset (Fig. 5), with results similarly found for the other datasets. Note that for this analysis the two universal parametrizations (univariate and multivariate) are used in order to compare the model performance with the well-established stability corrections.

- Air temperature: the model performance for different approaches for heat-flux parametrizations is generally robust to air temperatures. Modified Stearns provides acceptable heat-flux parametrizations for very low temperatures, whereas Holtslag provides better 

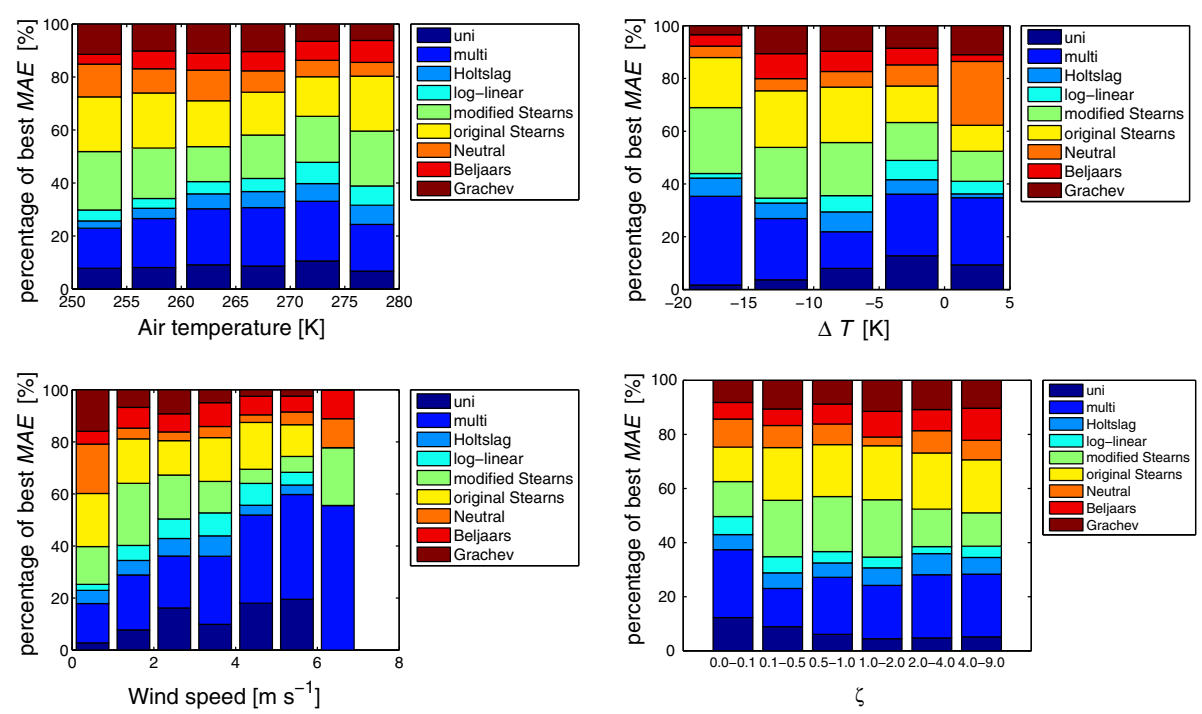

Fig. 5 Frequency in [\%] of the smallest $M A E$ value depending on the air temperature (upper left), temperature difference (upper right), wind speed (lower left) and stability parameter (lower right) for the WFJ11 dataset

results for temperatures above $260 \mathrm{~K}$. The univariate and multivariate parametrizations perform adequately in almost the whole range of air temperatures.

- Temperature difference: while original Stearns shows the optimum model performance for large temperature differences, the univariate parametrization has the optimum heat-flux estimates for low temperature differences. The newly developed multivariate parametrization is an acceptable alternative to original Stearns for large temperature differences.

- Wind speed: the univariate parametrization shows the largest percentage of optimum MAE values for moderate wind speeds $\left(2-5 \mathrm{~m} \mathrm{~s}^{-1}\right)$ and the performance of the multivariate parametrization is optimum for wind speeds $>5 \mathrm{~m} \mathrm{~s}^{-1}$.

- Stability parameter: the univariate parametrization performs optimum for near-neutral and weakly stable conditions. The results of the multivariate parametrization clearly improve in more stable conditions compared to the other approaches.

\section{Conclusion}

We tested existing and newly developed parametrizations to correct for atmospheric stability over snow, with turbulent heat-flux parametrizations improved by using two different approaches for a stability correction. We partitioned the error into a contribution from the bulk formulation and the model performance of the stability correction itself. We compared wellestablished stability corrections with two new stability corrections over snow and summarize the major findings as follows:

- Uncertainty in the bulk formulation: investigations have shown a modelled $M A E$ value of $6 \mathrm{~W} \mathrm{~m}^{-2}$ using non-parametrized $\psi$ values. This $M A E$ value corresponds to the accuracy of the bulk formulation itself and has to be tolerated in current physically-based models. In 
particular, large sensible heat fluxes contribute to the error because they are significantly underestimated in the model.

- Validation of well-established stability corrections: the optimum heat-flux parametrizations are those of Holtslag and De Bruin (1988) and Beljaars and Holtslag (1991) $\left(M A E: 7 \mathrm{~W} \mathrm{~m}^{-2}\right)$, followed by the simple log-linear approach $\left(M A E: 8 \mathrm{~W} \mathrm{~m}^{-2}\right)$, the stability correction of Grachev et al. (2007) and the stability correction of Stearns and Weidner (1993) (modified by Michlmayr et al. 2008) (MAE:11 $\mathrm{W} \mathrm{m}^{-2}$ ). A neutral boundary-layer assumption strongly overestimates heat fluxes towards the snow surface $\left(M A E: 22 \mathrm{~W} \mathrm{~m}^{-2}\right)$. All investigated stability corrections perform reasonably during low wind speeds and small temperature differences but show biases during high wind speeds.

- Uncertainty in the newly developed parametrizations: universal parametrizations for a univariate and multivariate approach have been developed herein. For the multivariate parametrization $M A E=7 \mathrm{~W} \mathrm{~m}^{-2}$ with a systematic bias of $4 \mathrm{~W} \mathrm{~m}^{-2}$; the approach of the univariate parametrization has a similar $M A E$ value and underestimates heat fluxes by $2 \mathrm{~W} \mathrm{~m}^{-2}$ on average. As none of the well-established parametrizations showed a reasonable performance for high wind speeds, our new multivariate parametrization presents itself as a viable alternative. In general the optimum performance was found for the multivariate parametrization with offset. The univariate parametrization could be an acceptable alternative for neutral atmospheric conditions and moderate wind speeds, and has additionally the advantage that only two empirical coefficients are required.

Stability corrections in general are affected by a large number of uncertainties. Many of these have not been systematically investigated in this study but could lead to a different performance of the stability corrections. The influence of a parametrization of the scalar roughness length, the uncertainty of the modelled stability parameter, uncertainties in the measurements of snow surface temperature and the measurement heights need further investigation to test the robustness of the results across all conditions.

Acknowledgements The work was funded by Swiss National Science Foundation (Project: Snow-atmosphere interactions driving snow accumulation and ablation in an Alpine catchment: The Dischma Experiment; SNFGrant: 200021_150146).

\section{Appendix}

We used the following stability corrections (see also "Electronic Supplement Material" in Sharan and Kumar (2011) for further discussion and additional stability corrections) (Table 6): 


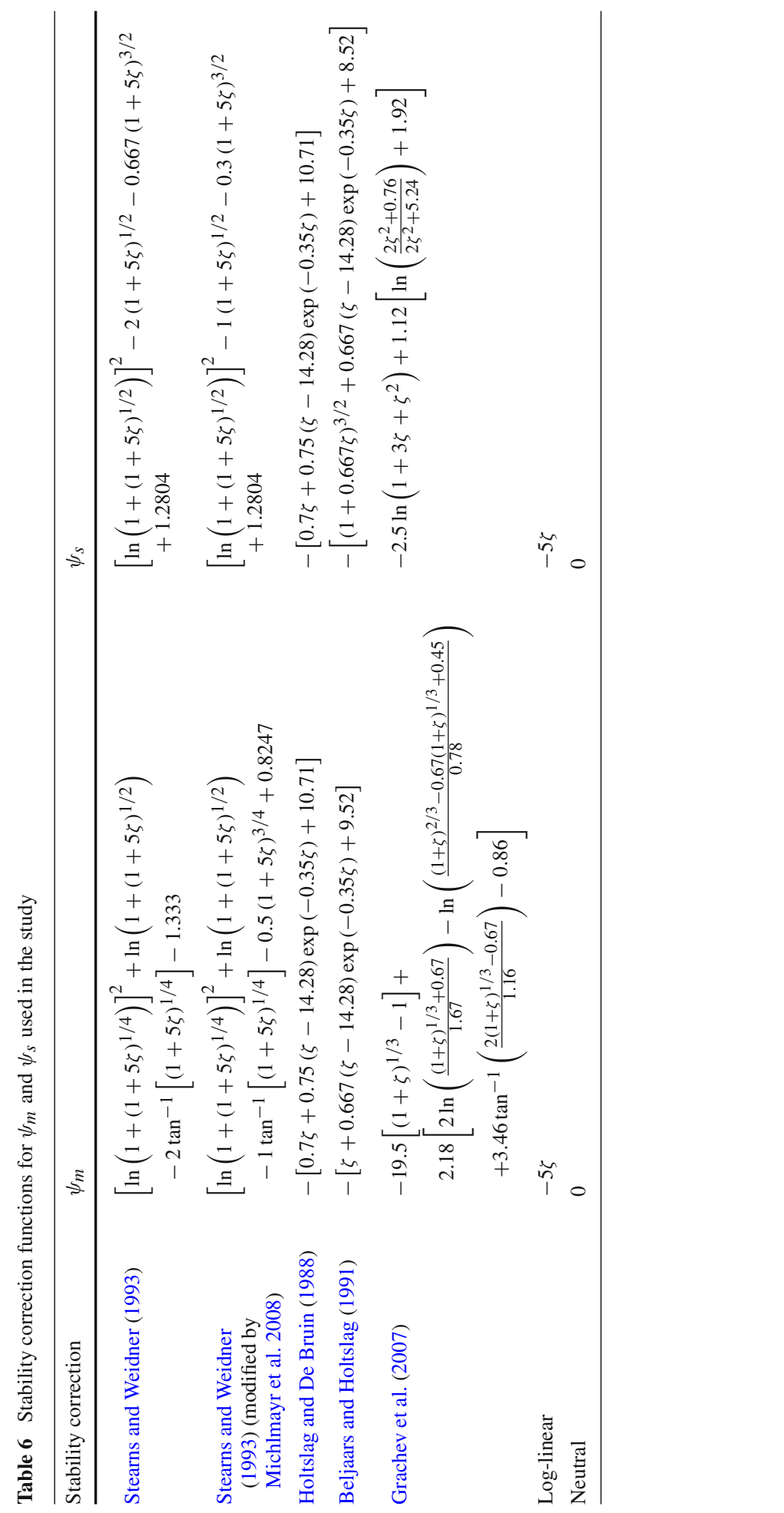




\section{References}

Andreas EL (1987) A theory for the scalar roughness and the scalar transfer coefficients over snow and sea ice. Boundary-Layer Meteorol 38:159-184

Andreas EL (2002) Parameterizing scalar transfer over snow and ice: a review. J Hydrometeorol 3:417-432

Andreas EL, Persson POG, Jordan RE, Horst TW, Guest PS, Grachev AA, Fairall CW (2010) Parameterizing turbulent exchange over sea ice in winter. J Hydrometeorol 11(1):87-104. doi:10.1175/2009JHM1102.1

Arck M, Scherer D (2002) Problems in the determination of sensible heat flux over snow. Geogr Ann 84 A(3-4):157-169

Beljaars ACM, Holtslag AAM (1991) Flux parametrization over land surfaces for atmospheric models. J Appl Meteorol 30:327-341

Blanc T (1987) Accuracy of bulk-method-determined flux, stability, and sea surface roughness. J Geophys Res Atmos 92:3867-3876

Bou-Zeid E, Higgins C, Huwald H, Meneveau C, Parlange MB (2010) Field study of the dynamics and modelling of subgridscale turbulence in a stable atmospheric surface layer over a glacier. J Fluid Mech 665:480-515

Conway JP, Cullen NJ (2016) Cloud effects on surface energy and mass balance in the ablation area of Brewster Glacier, New Zealand. Cryosphere 10(1):313-328. doi:10.5194/tc-10-313-2016

Cullen NJ, Steffen K (2001) Unstable near-surface boundary layer conditions in summer on top of the Greenland ice sheet. Geophys Res Lett 28:4491-4493. doi:10.1029/2001GL013417

Cullen NJ, Steffen K, Blanken PD (2007) Nonstationarity of turbulent heat fluxes at Summit, Greenland. Boundary-Layer Meteorol 122:439-455. doi:10.1007/s10546-006-9112-2

Cullen NJ, Mölg T, Conway J, Steffen K (2014) Assessing the role of sublimation in the dry snow zone of the Greenland ice sheet in a warming world. J Geophys Res Atmos 119:6563-6577. doi:10.1002/ 2014JD021557

Dadic R, Mott R, Lehning M, Carenzo M, Anderson B, Mackintosh A (2013) Sensitivity of turbulent fluxes to wind speed over snow surfaces in different climatic settings. Adv Water Resour 55:178-189

Dyer AJ (1974) A review of flux-profile relationships. Boundary-Layer Meteorol 7:363-372

Essery R, Granger R, Pomeroy J (2006) Boundary-layer growth and advection of heat over snow and soil patches: modelling and parameterization. Hydrol Process 20:953-967

Essery R, Morin S, Lejeune Y, Menard CB (2013) A comparison of 1701 snow models using observations from an alpine site. Adv Water Resour 55:131-148

Föhn P (1973) Short term snow melt and ablation derived from heat- and mass-balance measurements. J Glaciol 12(65):275-289

Funk M (1985) Räumliche Verteilung der Massenbilanz auf dem Rhonegletscher und ihre Beziehung zu Klimaelementen. Zürcher Geographische Schriften. 24:183 pp

Guo X, Yang K, Zhao L, Yang W, Li S, Zhu M, Yao T, Chen Y (2011) Critical evaluation of scalar roughness length parametrizations over a melting valley glacier. Boundary-Layer Meteorol 139:307-332

Grachev AA, Fairall CW, Persson POG, Andreas EL, Guest PS (2005) Stable boundary-layer scaling regimes: the SHEBA data. Boundary-Layer Meteorol 116(2):201-235. doi:10.1007/s10546-004-2729-0

Grachev AA, Andreas EL, Fairall CW, Guest PS, Persson POG (2007) SHEBA flux-profile relationships in the stable atmospheric boundary layer. Boundary-Layer Meteorol 124(3):315-333. doi:10.1007/s10546007-9177-6

Grachev AA, Andreas EL, Fairall CW, Guest PS, Persson POG (2013) The critical Richardson number and limits of applicability of local similarity theory in the stable boundary layer. Boundary-Layer Meteorol 147(1):51-82. doi:10.1007/s10546-012-9771-0

Högström U (1988) Non-dimensional wind and temperature profiles in the atmospheric surface layer: a reevaluation. Boundary-Layer Meteorol 42:55-78

Holtslag AAM, De Bruin HAR (1988) Applied modeling of the nighttime surface energy balance over land. J Appl Meteorol 27(6):689-704

Huwald H, Higgins CW, Boldi MO, Bou-Zeid E, Lehning M, Parlange MB (2009) Albedo effect on radiative errors in air temperature measurements. Water Resour Res 45:W08431. doi:10.1029/2008WR007600

Joffre SM (1982) Momentum and heat transfers in the surface layer over a frozen sea. Boundary-Layer Meteorol 24:211-229

Large WG, Pond S (1982) Sensible and latent heat flux measurements over the ocean. J Phys Oceanogr 11:324-336

Lehning M, Bartelt P, Brown B, Fierz C (2002) A physical SNOWPACK model for the Swiss avalanche warning: Part III: meteorological forcing, thin layer formation and evaluation. Cold Reg Sci Technol 35(3):169-184 
Marks D, Dozier J (1992) Climate and energy exchange at the snow surface in the Alpine region of the Sierra Nevada. 2. Snow cover energy balance. Water Resour Res 28(11):3043-3054

Martin E, Lejeune Y (1998) Turbulent fluxes above the snow surface. Ann Glaciol 26:179-183

Massmann WJ, Lee X (2002) Eddy covariance flux corrections and uncertainties in long-term studies of carbon and energy exchanges. Agric Forest Meteorol 113:121-144

Michlmayr G, Lehning M, Koboltschnig G, Holzmann H, Zappa M, Mott R, Schöner W (2008) Application of the Alpine 3D model for glacier mass balance and glacier runoff studies at Goldbergkees, Austria. Hydrol Process 22(19):3941-3949

Mott R, Egli L, Grünewald T, Dawes N, Manes C, Bavay M, Lehning M (2011) Micrometeorological processes driving snow ablation in an Alpine catchment. The Cryosphere 5:1083-1098

Mott R, Gromke C, Grünewald T, Lehning M (2013) Relative importance of advective heat transport and boundary layer decoupling in the melt dynamics of a patchy snow cover. Adv Water Resources 55:88-97

Mott R, Daniels M, Lehning M (2015) Atmospheric flow development and associated changes in turbulent sensible heat flux over a patchy mountain snow cover. J Hydrometeorol 16:1315-1340

Munro DS (1980) Exponential-linear stability correction functions for weak to moderate instability near the ground. Boundary-Layer Meteorol 19:125-131

Nishimura K, Nemoto M (2005) Blowing snow at Mizuho station, Antarctica. Phil Trans R Soc A 363:16471662

Obukhov AM (1946) Turbulence in an atmosphere with a non-uniform temperature. Trudy Inst Teoret Geophys Akad Nauk SSSR. 1:95-115 (translation in: Boundary-Layer Meteorol 1971. 2:7-29)

Plüss C, Mazzoni R (1994) The role of turbulent heat fluxes in the energy balance of high alpine snow cover. Nordic Hydrol 25:25-38

Pohl S, Marsh P, Liston GE (2006) Spatial-temporal variability in turbulent fluxes during spring snowmelt. Arct Antarct Alp Res 38:136-146

Rannik Ü, Vesala T (1999) Autoregressive filtering versus linear detrending in estimation of fluxes by the eddy covariance method. Boundary-Layer Meteorol 91:259-280

Sharan M, Kumar P (2011) Estimation of upper bounds for the applicability of non-linear similarity functions for non-dimensional wind and temperature profiles in the surface layer in very stable conditions. Proc $\mathrm{R}$ Soc A 467(2126):473-494. doi:10.1098/rspa.2010.0220

Smeets CJPP, van den Broeke MR (2008b) The parameterization of scalar transfer over rough ice. BoundaryLayer Meteorol 128(3):339-355. doi:10.1007/s10546-008-9292-z

Sorbjan Z (2010) Gradient-based scales and similarity laws in the stable boundary layer. QJR Meteorol Soc 136(650A):1243-1254. doi:10.1002/qj.638

Sorbjan Z (2016) Similarity scaling systems for stably stratified turbulent flows. QJR Meteorol Soc 142(695B):805-810. doi:10.1002/qj.2682

Stearns CR, Weidner GA (1993) Sensible and Latent heat flux estimates in Antarctica. Antarctic Research Series 61:109-138

Stössel F, Guala M, Fierz C, Manes C, Lehning M (2010) Micrometeorological and morphological observations of surface hoar dynamics on a mountain snow cover. Water Resources Res 46(4):W04511

Stull RB (1988) An introduction to boundary layer meteorology. Kluwer Acad Publishers, Dordrecht 666 pp

Vickers D, Mahrt L, Andreas EL (2015) Formulation of the sea surface friction velocity in terms of the mean wind and bulk stability. J Appl Meteor Climatol 54(3):691-703

Webb EK (1970) Profile relationships the log-linear range and extension to strong stability. QJR Meteorol Soc 96:67-90

Zeng X, Zhao M, Dickinson RE (1998) Intercomparison of bulk aerodynamic algorithms for the computation of sea surface fluxes using TOGA COARE and TAO data. J Clim 11:2628-2644 